\title{
ALTERNATING EULER SUMS AND SPECIAL VALUES OF THE WITTEN MULTIPLE ZETA FUNCTION ATTACHED TO $\mathfrak{s} \mathfrak{o}(5)$
}

\author{
JIANQIANG ZHAO
}

(Received 21 January 2010; accepted 23 December 2010)

Communicated by F. Calegari

\begin{abstract}
We study the Witten multiple zeta function associated with the Lie algebra $\mathfrak{s o}(5)$. Our main result shows that its special values at nonnegative integers are always expressible by alternating Euler sums. More precisely, every such special value of weight $w$ at least 2 is a finite $\mathbb{Q}$-linear combination of alternating Euler sums of weight $w$ and depth at most 2, except when the only nonzero argument is one of the two last variables, in which case $\zeta(w-1)$ is needed.
\end{abstract}

2010 Mathematics subject classification: primary 11M41; secondary 11S45, 17B20.

Keywords and phrases: Witten zeta functions, Witten volume formulas, alternating Euler sums.

\section{Introduction}

The Witten multiple zeta function associated with the Lie algebra $\mathfrak{s o ( 5 )}$ was defined by Komori, Matsumoto and Tsumura [8] as follows:

$$
\zeta_{\mathfrak{s o}(5)}\left(s_{1}, \ldots, s_{4}\right)=\sum_{m, n=1}^{\infty} \frac{1}{m^{s_{1}} n^{s_{2}}(m+n)^{s_{3}}(m+2 n)^{s_{4}}} .
$$

The sum converges provided that $\operatorname{Re}\left(s_{1}+s_{2}+s_{3}+s_{4}\right)>2, \operatorname{Re}\left(s_{1}+s_{3}+s_{4}\right)>1$, and $\operatorname{Re}\left(s_{2}+s_{3}+s_{4}\right)>1$. We call $s_{1}+s_{2}+s_{3}+s_{4}$ the weight. Essouabri [3] and Matsumoto [9] defined more general multiple zeta functions and studied their analytic continuations. However, the function in (1.1) already generalizes both the zeta function $\zeta_{\mathfrak{s o}(5)}(s, s, s, s)$ suggested by Zagier [17, Section 7] after Witten [16] and the MordellTornheim double zeta function [10,13] (see Section 2.2). Zagier and Garoufalidis

The author thanks the Max Planck Institute for Mathematics in Bonn for financial support during his sabbatical leave during 2009, when this work was done.

(C) 2011 Australian Mathematical Publishing Association Inc. 1446-7887/2011 \$16.00 
independently showed that, for every positive integer $m$, there is a rational number $c(m)$ such that

$$
\zeta_{\mathfrak{s o}(5)}(2 m, 2 m, 2 m, 2 m)=c(m) \times \pi^{8 m} \text {. }
$$

This had already been proposed by Witten from physical considerations for more general types of zeta functions associated with semisimple Lie algebras. Special values like those in (1.2) are the main objects of study here. We regard $\zeta_{\mathfrak{s o}(5)}\left(s_{1}, \ldots, s_{4}\right)$ as a special value when all the arguments $s_{1}, \ldots, s_{4}$ are nonnegative integers. Note that negative arguments are allowed in some other papers on special values.

In [15], Tsumura considered the special values of (1.1) at nonnegative integers. In particular, when the weight is an odd number he showed that the special values of (1.1) are $\mathbb{Q}$-linear combinations of products of Riemann zeta values at positive integers, with slightly stronger restrictions on the arguments than needed just to guarantee convergence. Since the function $\zeta_{\mathfrak{s o}(5)}\left(s_{1}, \ldots, s_{4}\right)$ has depth 2 , this type of result is commonly referred to as a 'parity relation'. For example, the (Euler-Zagier) multiple zeta value at positive integers, given by

$$
\zeta\left(s_{1}, \ldots, s_{d}\right):=\sum_{m_{1}>\cdots>m_{d} \geq 1} m_{1}^{-s_{1}} m_{2}^{-s_{2}} \cdots m_{d}^{-s_{d}},
$$

has the well-known property that if the weight is at least 2 , and the weight and the depth have different parities, then it may be written as a $\mathbb{Q}$-linear combination of products of multiple zeta values of lower depths (see [4, 14]). It is expected that, in general, when the weight is even and large enough, such relations may not always hold.

We investigate the convergent special values of $\zeta_{\mathfrak{s o}(5)}\left(s_{1}, \ldots, s_{4}\right)$ at nonnegative integers without any parity restriction on the weight. It turns out that they are closely related to alternating Euler sums (see Section 2.1). Here is our main result.

THEOREM 1.1. Let $s_{1}, \ldots, s_{4}$ be nonnegative integers such that $s_{1}+s_{3}+s_{4}>1$, $s_{2}+s_{3}+s_{4}>1$ and $w:=s_{1}+s_{2}+s_{3}+s_{4}>2$. Then $\zeta_{\mathfrak{s o}(5)}\left(s_{1}, \ldots, s_{4}\right)$ may be written as a finite $\mathbb{Q}$-linear combination of alternating Euler sums of weight $w$ and depths at most 2 , unless $s_{1}=s_{2}=s_{3}=0$ or $s_{1}=s_{2}=s_{4}=0$, in which cases $\zeta(w-1)$ is needed.

In [24], we considered the Witten multiple zeta function associated with the Lie algebra $\mathfrak{s l}(4)$. In general, we believe that not only multiple zeta values and alternating Euler sums, but also more general special values of multiple polylogarithms at roots of unity (see $[12,18,23]$ ) may be used to study many other types of Witten multiple zeta functions, such as those appearing in the recent work of Komori et al. [5, 6, 8]. This is investigated in detail elsewhere (see, in particular, [22]).

\section{Some preliminaries}

2.1. Alternating Euler sum. For positive integers $s_{1}, \ldots, s_{d}$, the alternating Euler sum is defined by

$$
\zeta\left(s_{1}, \ldots, s_{d} ; x_{1}, \ldots, x_{d}\right):=\sum_{m_{1}>\cdots>m_{d} \geq 1} \frac{x_{1}^{m_{1}} \cdots x_{d}^{m_{d}}}{m_{1}^{s_{1}} \cdots m_{d}^{s_{d}}},
$$


where $x_{j}= \pm 1$ when $1 \leq j \leq d$, and $\left(s_{1}, x_{1}\right) \neq(1,1)$. We call $s_{1}+\cdots+s_{d}$ the weight and $d$ the depth. To save space, we write $\zeta\left(t_{1}, \ldots, t_{d}\right)$, where each $t_{j}$ is $s_{j}$ if $x_{j}=1$ and $\bar{s}_{j}$ if $x_{j}=-1$. For example, we have the familiar result $\zeta(\overline{1})=\zeta(1 ;-1)=$ $-\log 2$, and the striking identity [21] that

$$
\zeta\left(\{3\}^{n}\right)=8^{n} \zeta\left(\{\overline{2}, 1\}^{n}\right),
$$

for every positive integer $n$, where $\{S\}^{n}$ means the string $S$ is repeated $n$ times. Identities like these, which are derived by (regularized) double shuffle relations, will be crucial in simplifying our computations in the last section of this paper.

2.2. Mordell-Tornheim zeta functions. These functions (see [10,13]) are given by

$$
\zeta_{\mathrm{MT}}\left(s_{1}, \ldots, s_{d} ; s\right)=\sum_{m_{1}, \ldots, m_{d}=1}^{\infty} m_{1}^{-s_{1}} m_{2}^{-s_{2}} \cdots m_{d}^{-s_{d}}\left(m_{1}+\cdots+m_{d}\right)^{-s} .
$$

Recently Bradley and Zhou [2, Theorem 2.2] showed that (2.2) converges absolutely if $\operatorname{Re}(s)+\sum_{j=1}^{\ell} \operatorname{Re}\left(s_{i_{j}}\right)>\ell$ for each nonempty subset $\left\{i_{1}, \ldots, i_{\ell}\right\}$ of $\{1,2, \ldots, d\}$. We may use the integral test and the well-known formula

$$
\sum_{m=1}^{n} m^{t}=\frac{1}{t+1}\left(B_{t+1}(n+1)-B_{t+1}(0)\right)
$$

where $B_{t+1}$ is the Bernoulli polynomial, to extend their proof to the following necessary and sufficient condition for convergence when all arguments are integers.

Proposition 2.1. Let $s_{1}, \ldots, s_{d}$ and $s$ be arbitrary integers. Then the MordellTornheim zeta function $\zeta_{\mathrm{MT}}\left(s_{1}, \ldots, s_{d} ; s\right)$ converges if and only if

$$
s+\sum_{j=1}^{\ell} s_{i_{j}}>\ell
$$

for each nonempty subset $\left\{i_{1}, \ldots, i_{\ell}\right\}$ of $\{1,2, \ldots, d\}$.

The following is a variation of [2, Theorem 1.1].

PROPOSITION 2.2. Let $s_{1}, \ldots, s_{d}$ and $s$ be nonnegative integers. If at most one of them is equal to 0 , then the Mordell-Tornheim zeta value $\zeta_{\mathrm{MT}}\left(s_{1}, \ldots, s_{d} ; s\right)$ may be expressed as a $\mathbb{Q}$-linear combination of multiple zeta values of the same weight and depth.

In this note, we will only need this proposition when the depth is 2 .

REMARK 2.3. In [2, Lemma 3.1], the arguments $s_{1}, s_{2}, \ldots$ are all positive integers. But the definition of Mordell-Tornheim zeta values in [2] only requires the arguments to be integers. A careful check of the proof of [2, Theorem 1.1] shows that 
Proposition 2.2 holds. However, [2, Theorem 1.1] is incompatible with general conjectures. For example, through easy manipulation we see that

$$
\zeta_{\mathrm{MT}}(0,0 ; 3)=\sum_{n=1}^{\infty} \sum_{m=1}^{\infty} \frac{1}{(m+n)^{3}}=\sum_{k=1}^{\infty} \sum_{l=1}^{k-1} \frac{1}{k^{3}}=\zeta(2)-\zeta(3)=\zeta(2)-\zeta(2,1),
$$

but we expect that $\zeta(2)$ cannot be written as a $\mathbb{Q}$-linear combination of multiple zeta values of depth 2 and weight 3 ; only one such value exists, namely $\zeta(2,1)=\zeta(3)$. In other words, we believe that $\zeta(2)$ is not a rational multiple of $\zeta(3)$.

2.3. Convergence domain of $\zeta_{\mathfrak{s o}(5)}\left(s_{1}, \ldots, s_{4}\right)$. In the following proposition, we consider only integer arguments, although it is not hard to extend it to complex variables. The result may be derived from the concrete singularity set given in [8], but the following proof is more straightforward.

Proposition 2.4. Let $s_{1}, \ldots, s_{4}$ be nonnegative integers. Then the sum

$$
\zeta_{\mathfrak{s o}(5)}\left(s_{1}, \ldots, s_{4}\right)=\sum_{m, n=1}^{\infty} \frac{1}{m^{s_{1}} n^{s_{2}}(m+n)^{s_{3}}(m+2 n)^{s_{4}}}
$$

converges if and only if

$$
s_{1}+s_{3}+s_{4}>1, \quad s_{2}+s_{3}+s_{4}>1 \text { and } s_{1}+s_{2}+s_{3}+s_{4}>2 .
$$

PROOF. First we observe that for all $m, n>0$,

$$
m+n<m+2 n<2(m+n) .
$$

Hence

$$
\begin{aligned}
\frac{1}{2^{s_{4}}} \zeta_{\mathrm{MT}}\left(s_{1}, s_{2} ; s_{3}+s_{4}\right) & =\sum_{m, n=1}^{\infty} \frac{1}{m^{s_{1}} n^{s_{2}}(m+n)^{s_{3}}(2 m+2 n)^{s_{4}}} \\
& \leq \sum_{m, n=1}^{\infty} \frac{1}{m^{s_{1}} n^{s_{2}}(m+n)^{s_{3}}(m+2 n)^{s_{4}}} \\
& \leq \sum_{m, n=1}^{\infty} \frac{1}{m^{s_{1}} n^{s_{2}}(m+n)^{s_{3}+s_{4}}} \\
& =\zeta_{\mathrm{MT}}\left(s_{1}, s_{2} ; s_{3}+s_{4}\right) .
\end{aligned}
$$

The proposition now follows immediately from the convergence criterion for the Mordell-Tornheim double zeta function in Proposition 2.1.

2.4. A combinatorial lemma. The following lemma (see [11, p. 48]) will be used often in the proof of Theorem 1.1. 
LEMMA 2.5. Let $s$ and t be positive integers, and let $x$ and $y$ be nonzero real numbers such that $x+y \neq 0$. Then

$$
\frac{1}{x^{s} y^{t}}=\sum_{a=0}^{s-1}\left(\begin{array}{c}
t+a-1 \\
a
\end{array}\right) \frac{1}{x^{s-a}(x+y)^{t+a}}+\sum_{b=0}^{t-1}\left(\begin{array}{c}
s+b-1 \\
b
\end{array}\right) \frac{1}{y^{t-b}(x+y)^{s+b}} .
$$

\section{Proof of Theorem 1.1}

We consider six mutually exclusive cases that together exhaust all the possibilities:

Case 1: $s_{4}=0$;

Case 2: $s_{2}>0, s_{3}>0$ and $s_{4}>0$;

Case 3: $s_{2}>0, s_{3}=0$ and $s_{4}>0$;

Case 4: $s_{1}>0, s_{2}=0$ and $s_{4}>0$;

Case 5: $s_{1}=s_{2}=s_{3}=0$ and $s_{4}>0$;

Case 6: $s_{1}=s_{2}=0, s_{3}>0$ and $s_{4}>0$.

Case 1. $s_{4}=0$. In this case, we have a Mordell-Tornheim double zeta value, which is handled by Proposition 2.2 unless $s_{1}=s_{2}=0$. If $s_{1}=s_{2}=0$, then, as long as $s \geq 3$,

$$
\zeta_{\mathfrak{s o}(5)}(0,0, s, 0)=\zeta(s, 0)=\sum_{m>n \geq 1} \frac{1}{m^{s}}=\sum_{m=1}^{\infty} \frac{m-1}{m^{s}}=\zeta(s-1)-\zeta(s) .
$$

Thus Theorem 1.1 is true in this case. This is the first of the two exceptional cases in which we need the Riemann zeta value with the weight lowered by 1 .

Case 2. $s_{2}, s_{3}, s_{4}>0$. Take $x=n$ and $y=m+n$ in Lemma 2.5; then

$$
\begin{aligned}
\zeta_{\mathfrak{s o}(5)}\left(s_{1}, \ldots, s_{4}\right)= & \sum_{a_{2}=0}^{s_{2}-1}\left(\begin{array}{c}
s_{3}+a_{2}-1 \\
a_{2}
\end{array}\right) \zeta_{\mathfrak{s o}(5)}\left(s_{1}, s_{2}-a_{2}, 0, s_{4}+s_{3}+a_{2}\right) \\
& +\sum_{a_{3}=0}^{s_{3}-1}\left(\begin{array}{c}
s_{2}+a_{3}-1 \\
a_{3}
\end{array}\right) \zeta_{\mathfrak{s o}(5)}\left(s_{1}, 0, s_{3}-a_{3}, s_{4}+s_{2}+a_{3}\right) .
\end{aligned}
$$

We recommend that the interested reader check the convergence of the above values from (2.3). The rule of thumb is that the convergence is automatic if we apply Lemma 2.5 when each $x_{j}$ is a positive combination of indices. In the following steps, we often omit this convergence checking, since it is straightforward in most cases. The only exception is (3.5).

Note that all the zeta values in (3.2) have the same weight, so the first sum of (3.2) leads us to Case 3 , since $s_{2}-a_{2}>0$; the second sum of (3.2) leads us to Case 4 if $s_{1}>0$ initially; and the second sum of (3.2) leads us to Case 5 if $s_{1}=0$ initially.

Case 3. $s_{3}=0$ and $s_{2}, s_{4}>0$. In this case,

$$
\zeta_{\mathfrak{s o}(5)}\left(s_{1}, s_{2}, 0, s_{4}\right)=\sum_{m, n=1}^{\infty} \frac{2^{s_{2}}}{m^{s_{1}}(2 n)^{s_{2}}(m+2 n)^{s_{4}}}=\sum_{m, n=1}^{\infty} \frac{2^{s_{2}-1}\left(1+(-1)^{n}\right)}{m^{s_{1}} n^{s_{2}}(m+n)^{s_{4}}} .
$$


Breaking this into two parts and applying Lemma 2.5 with $x=m$ and $y=n$ to the second part, we deduce that the right-hand side of (3.3) is equal to

$$
\begin{aligned}
2^{s_{2}-1} & \left\{\zeta_{\mathrm{MT}}\left(s_{1}, s_{2} ; s_{4}\right)+\sum_{a_{1}=0}^{s_{1}-1}\left(\begin{array}{c}
s_{2}+a_{1}-1 \\
a_{1}
\end{array}\right) \sum_{m, n=1}^{\infty} \frac{(-1)^{n}}{m^{s_{1}-a_{1}}(m+n)^{s_{4}+s_{2}+a_{1}}}\right. \\
+ & \left.\sum_{a_{2}=0}^{s_{2}-1}\left(\begin{array}{c}
s_{1}+a_{2}-1 \\
a_{2}
\end{array}\right) \sum_{m, n=1}^{\infty} \frac{(-1)^{n}}{n^{s_{2}-a_{2}}(m+n)^{s_{4}+s_{1}+a_{2}}}\right\} \\
= & 2^{s_{2}-1}\left\{\zeta_{\mathrm{MT}}\left(s_{1}, s_{2} ; s_{4}\right)+\sum_{a_{1}=0}^{s_{1}-1}\left(\begin{array}{c}
s_{2}+a_{1}-1 \\
a_{1}
\end{array}\right) \zeta\left(\overline{s_{4}+s_{2}+a_{1}}, \overline{s_{1}-a_{1}}\right)\right. \\
& \left.+\sum_{a_{2}=0}^{s_{2}-1}\left(\begin{array}{c}
s_{1}+a_{2}-1 \\
a_{2}
\end{array}\right) \zeta\left(s_{4}+s_{1}+a_{2}, \overline{s_{2}-a_{2}}\right)\right\} .
\end{aligned}
$$

Observe that the last component of every alternating Euler sum (or double zeta value) above is positive and the weights do not change. So Theorem 1.1 holds in this case.

Case 4. $s_{1}>0, s_{2}=0$ and $s_{1}, s_{4}>0$. Take $x=m$ and $y=m+2 n$ in Lemma 2.5; since

$$
\zeta_{\mathfrak{s o}(5)}\left(s_{1}, 0, s_{3}, s_{4}\right)=\sum_{m, n=1}^{\infty} \frac{1}{m^{s_{1}}(m+n)^{s_{3}}(m+2 n)^{s_{4}}},
$$

we find that $\zeta_{\mathfrak{s o}(5)}\left(s_{1}, 0, s_{3}, s_{4}\right)$ is equal to

$$
\begin{aligned}
& \sum_{a_{1}=0}^{s_{1}-1}\left(\begin{array}{c}
s_{4}+a_{1}-1 \\
a_{1}
\end{array}\right) \frac{1}{2^{s_{4}+a_{1}}} \zeta\left(s_{3}+s_{4}+a_{1}, s_{1}-a_{1}\right) \\
& \quad+\sum_{a_{4}=0}^{s_{4}-1}\left(\begin{array}{c}
s_{1}+a_{4}-1 \\
a_{4}
\end{array}\right) \frac{1}{2^{s_{1}+a_{4}}} \zeta_{\mathfrak{s o}(5)}\left(0,0, s_{3}+s_{1}+a_{4}, s_{4}-a_{4}\right) .
\end{aligned}
$$

Note that all the double zeta values in (3.4) have the same weight as when we started. We remind the reader that to determine the weight of a multiple zeta value, it is not enough just to add all the components to see that the weight does not change. We also need to check that every component is positive, and in this particular case, that the last components $s_{1}-a_{1}$ and $s_{4}-a_{4}$ are positive. This is guaranteed by the range of summation of $a_{1}$ and $a_{4}$. Since $s_{1}>0$ and $s_{4}>0$, we are reduced to Case 6 .

Case 5. $s_{1}=s_{2}=s_{3}=0$ and $s_{4}>0$. Write $s=s_{4} \geq 3$ (for convergence); then

$$
\begin{aligned}
\zeta_{\mathfrak{s o}(5)}(0,0,0, s) & =\sum_{m, n=1}^{\infty} \frac{1}{(m+2 n)^{s}}=\frac{1}{2} \sum_{m, n=1}^{\infty} \frac{1+(-1)^{n}}{(m+n)^{s}} \\
& =\frac{1}{2}\left\{\zeta(s, 0)+\sum_{k>m \geq 1} \frac{(-1)^{m+k}}{k^{s}}\right\} .
\end{aligned}
$$


Now the sum over $m$ is 0 unless $k$ is even, so by (3.1),

$$
\begin{aligned}
\zeta_{\mathfrak{s o}(5)}(0,0,0, s) & =\frac{1}{2}\left\{\zeta(s-1)-\zeta(s)+\sum_{2 k \geq 1} \frac{-1}{(2 k)^{s}}\right\} \\
& =\frac{1}{2}\left\{\zeta(s-1)-\zeta(s)-\frac{1}{2^{s}} \zeta(s)\right\} .
\end{aligned}
$$

Thus Theorem 1.1 holds in this case. This is the second exceptional case in which we need the Riemann zeta value with the weight lowered by 1 .

Case 6. $s_{1}=s_{2}=0, s_{3}>0$ and $s_{4}>0$. Write $r=s_{3}$ and $t=s_{4}$; then $r+t \geq 3$ (for convergence). Take $x=-m-n$ and $y=m+2 n$ in Lemma 2.5; then $\zeta_{\mathfrak{s o}(5)}(0,0, r, t)$ is equal to

$$
\begin{aligned}
\sum_{m, n=1}^{\infty} & \frac{(-1)^{r}}{(-m-n)^{r}(m+2 n)^{t}} \\
= & \sum_{a=0}^{r-2}\left(\begin{array}{c}
t+a-1 \\
a
\end{array}\right) \sum_{m, n=1}^{\infty} \frac{(-1)^{a}}{n^{t+a}(m+n)^{r-a}} \\
& +\sum_{a=0}^{t-2}\left(\begin{array}{c}
r+a-1 \\
a
\end{array}\right) \sum_{m, n=1}^{\infty} \frac{(-1)^{r}}{n^{r+a}(m+2 n)^{t-a}} \\
& +(-1)^{r}\left(\begin{array}{c}
t+r-2 \\
r-1
\end{array}\right) \sum_{m, n=1}^{\infty}\left(\frac{1}{n^{r+t-1}(m+2 n)}-\frac{1}{n^{r+t-1}(m+n)}\right)
\end{aligned}
$$

The inner infinite sum of the first sum is exactly $(-1)^{a} \zeta(r-a, t+a)$, while the second sum may be dealt with by the same method as (3.3). Indeed, if $u$ and $v$ are positive integers and $v>1$, then

$$
\sum_{m, n=1}^{\infty} \frac{1}{n^{u}(m+2 n)^{v}}=2^{u-1} \sum_{m, n=1}^{\infty} \frac{1+(-1)^{n}}{n^{u}(m+n)^{v}}=2^{u-1}\{\zeta(v, u)+\zeta(v, \bar{u})\} .
$$

Note that all the alternating Euler sums in (3.5) have the same weight (and the second components are all positive, since $r, t>0$ ). So we only need to consider the last infinite sum. Assume that $s=r+t-1>1$, and define $S^{(N)}$ to be

$$
\begin{aligned}
\sum_{m, n=1}^{N}\left(\frac{1}{n^{s}(m+2 n)}-\frac{1}{n^{s}(m+n)}\right) & =\sum_{n=1}^{N} \frac{1}{n^{s}}\left(\sum_{m=1+2 n}^{N+2 n}-\sum_{m=1+n}^{N+n}\right) \frac{1}{m} \\
& =\sum_{n=1}^{N} \frac{1}{n^{s}}\left(\sum_{m=N+n+1}^{N+2 n}+\sum_{m=1}^{n}-\sum_{m=1}^{2 n}\right) \frac{1}{m} .
\end{aligned}
$$

Noting that $s>1$ and therefore

$$
\sum_{n=1}^{N} \frac{1}{n^{s}} \sum_{m=N+n+1}^{N+2 n} \frac{1}{m}<\sum_{n=1}^{N} \frac{1}{n^{s-1} N} \ll \log N / N \rightarrow 0 \quad \text { as } N \rightarrow \infty,
$$


we see quickly that

$$
\begin{aligned}
\lim _{N \rightarrow \infty} S^{(N)} & =\sum_{n \geq m \geq 1} \frac{1}{n^{s} m}-\sum_{2 n \geq m \geq 1} \frac{1}{n^{s} m} \\
& =\zeta(s+1)+\zeta(s, 1)-2^{s-1} \sum_{n \geq m \geq 1} \frac{1+(-1)^{n}}{n^{s} m} \\
& =\left(1-2^{s-1}\right)(\zeta(s+1)+\zeta(s, 1))-2^{s-1}(\zeta(\bar{s}, 1)+\zeta(\overline{s+1}))
\end{aligned}
$$

where all the Euler sums have the same weight. This concludes the proof of Theorem 1.1.

REMARK 3.1. Note that Theorem 1.1 does not imply Tsumura's result about odd weight values, since the parity relations do not hold in general for alternating Euler sums. For example, we know that the $\mathbb{Q}$-linear space generated by the Riemann zeta values of weight 3 has dimension one, since $\zeta(3)=\zeta(2,1)$. Broadhurst conjectures that the dimension $F_{n}$ of the space generated by weight $n$ alternating Euler sums over $\mathbb{Q}$ is a Fibonacci number: $F_{1}=1, F_{2}=2, F_{3}=3, F_{4}=5$, and so on. It is easy to verify [19] that the weight-three space is spanned by $\zeta(3), \zeta(\overline{1}, 2)$ and $\zeta(\overline{1}, 1,1)$. Note also that the depth-one subspace is generated by $\zeta(3)$ since $\zeta(\overline{3})=-\frac{3}{4} \zeta(3)$. Therefore Broadhurst's conjecture suggests that the alternating Euler sum $\zeta(\overline{1}, 2)$ cannot be reduced to depth 1 . Since $\zeta(\overline{1}, 2)$ has odd weight and even depth the parity relation does not hold for it.

\section{Some examples and a conjecture}

Finally, we present some numerical examples, and propose a conjecture on the space generated by the special values of $\zeta_{\mathfrak{s o}(5)}$. In [15], Tsumura found some values of $\zeta_{\mathfrak{s o}(5)}\left(s_{1}, \ldots, s_{4}\right)$ when the weight is odd. Using our general approach, we may compute all the convergent values, and in particular we are able to confirm all the odd weight values of $\zeta_{\mathfrak{s o}(5)}$ in [14]. In practice, one may first convert our formulas to computer programs and then compute with MAPLE. As a safeguard, we checked all the equations in this section numerically using EZface [1]. In what follows, we only consider the regular cases in each weight, that is, we exclude the two exceptional cases.

We first list all the regular weight-three values:

$$
\begin{aligned}
& \zeta_{\mathfrak{s o}(5)}(0,0,1,2)=-\frac{1}{2} \zeta(3)+3 \zeta(\overline{1}, 2), \\
& \zeta_{\mathfrak{s o}(5)}(0,1,0,2)=\frac{5}{4} \zeta(3)-3 \zeta(\overline{1}, 2), \\
& \zeta_{\mathfrak{s o}(5)}(1,0,0,2)=\frac{3}{2} \zeta(\overline{1}, 2)+\frac{1}{16} \zeta(3), \\
& \zeta_{\mathfrak{s o}(5)}(0,0,2,1)=\frac{1}{4} \zeta(3), \\
& \zeta_{\mathfrak{s o}(5)}(0,1,2,0)=\zeta(3),
\end{aligned}
$$




$$
\begin{aligned}
& \zeta_{\mathfrak{s o}(5)}(1,0,2,0)=\zeta(3), \\
& \zeta_{\mathfrak{s o}(5)}(0,1,1,1)=\frac{3}{4} \zeta(3), \\
& \zeta_{\mathfrak{s o}(5)}(1,0,1,1)=\frac{5}{8} \zeta(3), \\
& \zeta_{\mathfrak{s o}(5)}(1,1,0,1)=\frac{11}{8} \zeta(3), \\
& \zeta_{\mathfrak{s o}(5)}(1,1,1,0)=2 \zeta(3) .
\end{aligned}
$$

Note that the first three values do not satisfy the conditions of Tsumura's theorem [14], so there is no contradiction with his result, even if Broadhurst's conjecture suggests that they cannot be rational multiples of $\zeta(3)$. However, $\zeta_{\mathfrak{s o}(5)}(0,1,1,1)=\frac{3}{4} \zeta(3)$ does not satisfy the conditions either, but it is reduced to depth 1 . It would be interesting to find out exactly when special values of $\zeta_{\mathfrak{s o}(5)}$ reduce to Riemann zeta values.

REMARK 4.1. The smallest weight of $\zeta_{\mathfrak{s o}(5)}\left(s_{1}, s_{2}, s_{3}, s_{4}\right)$ to be considered is 3 because of the convergence constraint (2.3). We have listed all the possible regular weight-three values above, and it seems that they do not span the whole weightthree space over $\mathbb{Q}$, because the whole space should have dimension 3 according to Broadhurst's conjecture. In fact, this was expected because $\zeta_{\mathfrak{s o}(5)}\left(s_{1}, s_{2}, s_{3}, s_{4}\right)$ only has depth 2, while all the depth-one and two alternating Euler sums only generate a proper subspace of dimension 2 over $\mathbb{Q}$, and this subspace may be generated by $\zeta(3)$ and $\zeta(\overline{1}, 2)$. We believe that this phenomenon happens in general for higher weights.

Conjecture 4.2. Let $w$ be a positive integer greater than 2 . Let $V_{w}$ be the $\mathbb{Q}$-vector space spanned by all the special values of $\zeta_{\mathfrak{s o}(5)}\left(s_{1}, s_{2}, s_{3}, s_{4}\right)$ of weight $w$, where $s_{1}, s_{2}, s_{3}$ and $s_{4}$ are nonnegative integers satisfying $s_{1}+s_{2}+s_{3}+s_{4}>0$, $s_{1}+s_{2}+s_{3}>0$ and $s_{1}+s_{2}+s_{4}>0$. Then $V_{w}$ coincides with the $\mathbb{Q}$-vector space spanned by all the alternating Euler sums of weight $w$ and depth at most 2 .

We have verified this conjecture for all weights up to 5 .

We now list all the 25 regular values of weight 4 :

$$
\begin{aligned}
& \zeta_{\mathfrak{s o}(5)}(0,0,1,3)=-\frac{7}{12} \zeta(4)+\frac{7}{3} \zeta(\overline{1}, 3) \\
& \zeta_{\mathfrak{s o}(5)}(0,1,0,3)=\frac{17}{24} \zeta(4)-\frac{7}{3} \zeta(\overline{1}, 3) \\
& \zeta_{\mathfrak{s o}(5)}(1,0,0,3)=-\frac{19}{96} \zeta(4)+\frac{7}{6} \zeta(\overline{1}, 3)-\frac{1}{2} \zeta(\overline{3}, 1) \\
& \zeta_{\mathfrak{s o}(5)}(0,0,3,1)=-\frac{1}{4} \zeta(4)+4 \zeta(\overline{3}, 1) \\
& \zeta_{\mathfrak{s o}(5)}(0,1,3,0)=\frac{1}{4} \zeta(4) \\
& \zeta_{\mathfrak{s o}(5)}(1,0,3,0)=\frac{1}{4} \zeta(4) \\
& \zeta_{\mathfrak{s o}(5)}(0,0,2,2)=\frac{3}{8} \zeta(4)-4 \zeta(\overline{3}, 1) \\
& \zeta_{\mathfrak{s o}(5)}(0,2,0,2)=\frac{1}{8} \zeta(4)+4 \zeta(\overline{3}, 1) \\
& \zeta_{\mathfrak{s o}(5)}(2,0,0,2)=\frac{9}{32} \zeta(4)
\end{aligned}
$$




$$
\begin{aligned}
& \zeta_{\mathfrak{s o}(5)}(0,2,2,0)=\frac{3}{4} \zeta(4), \\
& \zeta_{\mathfrak{s o}(5)}(2,0,2,0)=\frac{3}{4} \zeta(4), \\
& \zeta_{\mathfrak{s o}(5)}(2,2,0,0)=\frac{5}{2} \zeta(4), \\
& \zeta_{\mathfrak{s o}(5)}(0,1,1,2)=\frac{1}{8} \zeta(4), \\
& \zeta_{\mathfrak{s o}(5)}(1,0,1,2)=\frac{3}{16} \zeta(4)-\zeta(\overline{3}, 1), \\
& \zeta_{\mathfrak{s o}(5)}(1,1,0,2)=\frac{5}{16} \zeta(4)-\zeta(\overline{3}, 1), \\
& \zeta_{\mathfrak{s o}(5)}(0,1,2,1)=\frac{1}{2} \zeta(4)-4 \zeta(\overline{3}, 1), \\
& \zeta_{\mathfrak{s o}(5)}(1,0,2,1)=2 \zeta(\overline{3}, 1), \\
& \zeta_{\mathfrak{s o}(5)}(1,1,2,0)=\frac{1}{2} \zeta(4), \\
& \zeta_{\mathfrak{s o}(5)}(0,2,1,1)=\frac{1}{4} \zeta(4)+4 \zeta(\overline{3}, 1), \\
& \zeta_{\mathfrak{s o}(5)}(1,2,0,1)=\frac{3}{4} \zeta(4)+2 \zeta(\overline{3}, 1), \\
& \zeta_{\mathfrak{s o}(5)}(1,2,1,0)=\frac{5}{4} \zeta(4), \\
& \zeta_{\mathfrak{s o}(5)}(2,0,1,1)=\frac{3}{8} \zeta(4)+\zeta(\overline{3}, 1), \\
& \zeta_{\mathfrak{s o}(5)}(2,1,0,1)=\frac{7}{8} \zeta(4)-\zeta(\overline{3}, 1), \\
& \zeta_{\mathfrak{s o}(5)}(2,1,1,0)=\frac{5}{4} \zeta(4), \\
& \zeta_{\mathfrak{s o}(5)}(1,1,1,1)=\frac{1}{2} \zeta(4)-2 \zeta(\overline{3}, 1) \text {. }
\end{aligned}
$$

There are 46 regular values of weight 5 and 74 regular values of weight 6 . Here are some interesting weight-six values:

$$
\begin{aligned}
& \zeta_{\mathfrak{s o}(5)}(0,2,2,2)=\frac{1}{105} \zeta(2)^{3}=\frac{1}{22680} \pi^{6}=0.04238929428 \ldots \\
& \zeta_{\mathfrak{s o}(5)}(2,0,2,2)=\frac{1}{210} \zeta(2)^{3}+\frac{3}{8} \zeta(3,3)-\frac{2}{3} \zeta(\overline{3}, 3)=0.03772580207 \ldots \\
& \zeta_{\mathfrak{s o}(5)}(2,2,0,2)=\frac{4}{105} \zeta(2)^{3}-\frac{3}{8} \zeta(3,3)+\frac{2}{3} \zeta(\overline{3}, 3)=0.15302602205 \ldots \\
& \zeta_{\mathfrak{s o}(5)}(2,2,2,0)=\zeta_{\mathrm{MT}}(2,2 ; 2)=\frac{8}{105} \zeta(2)^{3}=\frac{1}{2835} \pi^{6}=0.3391143543 \ldots \\
& \zeta_{\mathfrak{s o}(5)}(1,1,2,2)=\frac{1}{84} \zeta(2)^{3}-\frac{3}{8} \zeta(3,3)+\frac{2}{3} \zeta(\overline{3}, 3)=0.0364554628649 \ldots \\
& \zeta_{\mathfrak{s o}(5)}(1,2,1,2)=\frac{3}{140} \zeta(2)^{3}-\frac{3}{8} \zeta(3,3)+\frac{2}{3} \zeta(\overline{3}, 3)=0.0788447571142 \ldots \\
& \zeta_{\mathfrak{s o}(5)}(2,1,1,2)=\frac{1}{60} \zeta(2)^{3}=\frac{1}{12960} \pi^{6}=0.07418126500 \ldots \\
& \zeta_{\mathfrak{s o}(5)}(1,2,2,1)=\frac{1}{30} \zeta(2)^{3}-\frac{3}{4} \zeta(3,3)+\frac{4}{3} \zeta(\overline{3}, 3)=0.1153002199792 \ldots \\
& \zeta_{\mathfrak{s o}(5)}(2,1,2,1)=\frac{3}{140} \zeta(2)^{3}+\frac{3}{8} \zeta(3,3)-\frac{2}{3} \zeta(\overline{3}, 3)=0.1119070670077 \ldots \\
& \zeta_{\mathfrak{s o}(5)}(2,2,1,1)=\frac{23}{420} \zeta(2)^{3}-\frac{3}{8} \zeta(3,3)+\frac{2}{3} \zeta(\overline{3}, 3)=0.2272072869870 \ldots
\end{aligned}
$$

Finally, we return to the question about Zagier's original version of Witten's zeta function attached to $\mathfrak{s o}(5)$. We would like to know the rational coefficients in (1.2), 
and the following formulas provide the first four such values:

$$
\begin{aligned}
\zeta_{\mathfrak{s o}(5)}(2,2,2,2) & =\frac{3}{700} \zeta(2)^{4}=\frac{2 \times 3}{5 \times 9 !} \pi^{8}, \\
\zeta_{\mathfrak{s o}(5)}(4,4,4,4) & =\frac{4311}{297797500} \zeta(2)^{8}=\frac{2^{5} \times 479}{5 \times 17 !} \pi^{16}, \\
\zeta_{\mathfrak{s o}(5)}(6,6,6,6) & =\frac{2490861}{45593675752625} \zeta(2)^{12}=\frac{2^{7} \times 5 \times 43 \times 19309}{3^{2} \times 7 \times 13 \times 23 !} \pi^{24}, \\
\zeta_{\mathfrak{s o}(5)}(8,8,8,8) & =\frac{138835874547}{670007833199392187500} \zeta(2)^{16} \\
& =\frac{2^{8} \times 13 \times 241 \times 64009163}{5 \times 17 \times 31 !} \pi^{32} .
\end{aligned}
$$

A complicated formula for $\zeta_{\mathfrak{s o}(5)}(2 m, 2 m, 2 m, 2 m)$ may be found by analytical methods (see [7]) or algebraic methods (see [20]). This formula implies that $c(m)$ is equal to

$$
\begin{aligned}
& \frac{2^{8 m-3}}{(8 m) !} \sum_{v=0}^{m} B_{2 v} B_{8 m-2 v}\left(\begin{array}{c}
8 m \\
2 v
\end{array}\right)\left\{\sum_{\mu=0}^{2 m-1}\left(\frac{2^{2 v-1}}{2^{2 m+\mu}}-(-1)^{\mu}\right)\right. \\
& \quad \times\left(\begin{array}{c}
4 m-\mu-2 \\
2 m-1
\end{array}\right)\left(\begin{array}{c}
2 m-2 v+\mu \\
2 m-2 v
\end{array}\right) \\
& \left.\quad+\sum_{\mu=0}^{2 m-2 v}\left(\frac{1}{2^{2 m+\mu}}+(-1)^{\mu}\right)\left(\begin{array}{c}
4 m-2 v-\mu-1 \\
2 m-1
\end{array}\right)\left(\begin{array}{c}
2 m-1+\mu \\
2 m-1
\end{array}\right)\right\},
\end{aligned}
$$

where the $B_{2 v}$ are Bernoulli numbers. So we have come full circle, back to the values with which we began, but now the rational coefficients of the powers of $\pi$ are determined precisely.

\section{References}

[1] J. Borwein, P. Lisonek and P. Irvine, 'An interface for evaluation of Euler sums', available online at http://oldweb.cecm.sfu.ca/cgi-bin/EZFace/zetaform.cgi.

[2] D. M. Bradley and X. Zhou, 'On Mordell-Tornheim sums and multiple zeta values', Ann. Sci. Math. Québec 34 (2010), 15-23.

[3] D. Essouabri, 'Singularités des séries de Dirichlet associées à des polynômes de plusieurs variables et applications en théorie analytique des nombres', Ann. Inst. Fourier (Grenoble) 47 (1997), 429-483.

[4] K. Ihara, M. Kaneko and D. Zagier, 'Derivation and double shuffle relations for multiple zeta values', Compositio Math. 142 (2006), 307-338.

[5] Y. Komori, K. Matsumoto and H. Tsumura, 'On Witten multiple zeta-functions associated with semisimple Lie algebras III', Proc. Conf. on Multiple Dirichlet Series and Applications to Automorphic Forms, Edinburgh, 2008, to appear. Preprint, arxiv: 0907.0955.

[6] Y. Komori, K. Matsumoto and H. Tsumura, 'Zeta and $L$-functions and Bernoulli polynomials of root systems', Proc. Japan Acad. Ser. A 84 (2008), 57-62. 
[7] Y. Komori, K. Matsumoto and H. Tsumura, 'Functional equations for zeta-functions of root systems', in: Number Theory: Dreaming in Dreams, (Osaka, 2008) (eds. T. Aoki et al.) (World Scientific, Singapore, 2009), pp. 135-183.

[8] Y. Komori, K. Matsumoto and H. Tsumura, 'On Witten multiple zeta-functions associated with semisimple Lie algebras II', J. Math. Soc. Japan 62 (2010), 355-394.

[9] K. Matsumoto, 'On Mordell-Tornheim and other multiple zeta-functions', in: Proc. of the Session in Analytic Number Theory and Diophantine Equations, Bonner Math. Schriften, 360 (2003), article number 25,17 pages.

[10] L. J. Mordell, 'On the evaluation of some multiple series', J. Lond. Math. Soc. 33 (1958), 368-371.

[11] N. Nielsen, Die Gammafunktion. Band I. Handbuch der Theorie der Gammafunktion. Band II. Theorie des Integrallogarithmus und verwandter Transzendenten (Chelsea, New York, 1965).

[12] T. Terasoma, 'Rational convex cones and cyclotomic multiple zeta values', Preprint, arXiv: math/0410306.

[13] L. Tornheim, 'Harmonic double series', Amer. J. Math. 72 (1950), 303-314.

[14] H. Tsumura, 'Combinatorial relations for Euler-Zagier sums', Acta Arith. 111 (2004), $27-42$.

[15] H. Tsumura, 'On Witten's type of zeta values attached to SO(5)', Arch. Math. (Basel) 82 (2004), $147-152$.

[16] E. Witten, 'On quantum gauge theories in two-dimensions', Comm. Math. Phys. 141 (1991), 153-209.

[17] D. Zagier, Values of zeta function and their applications, in: Proc. of the First European Congress of Math. Vol. 2 (Birkhäuser, Basel-Boston-Berlin, 1994), pp. 497-512.

[18] J. Zhao, 'Multiple polylogarithm values at roots of unity', C. R. Acad. Sci. Paris Ser. I 346 (2008), 1029-1032.

[19] J. Zhao, 'Integral structures of multi-polylogs at roots of unity', Preprint, 2010.

[20] J. Zhao, 'Witten volume formulas for semi-simple Lie algebras', Integers, arXiv:1001.3630, in press.

[21] J. Zhao, 'On a conjecture of Borwein, Bradley and Broadhurst', J. Reine Angew. Math. 639 (2010), 223-233.

[22] J. Zhao, 'Multi-polylogs at twelfth roots of unity and special values of Witten multiple zeta function attached to the exceptional Lie algebra $\mathfrak{g}_{2}$ ', J. Algebra Appl. 9 (2010), 327-337.

[23] J. Zhao, 'Standard relations of multiple polylogarithm values at roots of unity', Doc. Math. 15 (2010), 1-34.

[24] J. Zhao and X. Zhou, 'Witten multiple zeta values attached to sl(4)', Tokyo J. Math., Preprint MPIM2009-41, 2009, arXiv:0903.2383, in press.

JIANQIANG ZHAO, Department of Mathematics, Eckerd College, St Petersburg FL 33711, USA e-mail: zhaoj@eckerd.edu 